\title{
Influência da temperatura na reprodução e longevidade do predador Orius thyestes Herring (Hemiptera, Anthocoridae)
}

\author{
Lívia Mendes Carvalhoํ, Vanda Helena Paes Bueno ${ }^{1} \&$ Simone Martins Mendes ${ }^{1}$
} 'Departamento de Entomologia, Universidade Federal de Lavras, Caixa Postal 3037, CEP 37200-000 Lavras, MG.
carvalholm@hotmail.com, vhpbueno@ufla.br

\begin{abstract}
Influence of the temperature on reproduction and longevity of the predator Orius thyestes Herring (Hemiptera, Anthocoridae). The development time and the fecundity of Orius species are strongly influenced by a wide range of factors, like environmental conditions and in particular temperature. This work aimed to evaluate the reproduction and adult longevity of Orius thyestes Herring, 1966 in different temperatures. Eggs of Anagasta kuehniella (Zeller, 1879) was provide as food supply. The experiment was carried out in climatic chambers at temperatures $16,19,22,25$, 28 and $31 \pm 1{ }^{\circ} \mathrm{C}$, RH $70 \pm 10 \%$ and photophase $12 \mathrm{~h}$. A deleterious effect of the temperature on $O$. thyestes was found at $16^{\circ} \mathrm{C}$, in which only $40 \%$ of the nymphs reached adulthood, of which only $19 \%$ presented normal morphological characteristics, without any alterations. The longest pre-oviposition period was found at $19^{\circ} \mathrm{C}(17.8$ days). The highest fecundities were found at 25 and $28^{\circ} \mathrm{C}\left(109.2\right.$ and $128.2 \mathrm{eggs} /$ female, respectively) and the lowest at $19{ }^{\circ} \mathrm{C}(22.8 \mathrm{eggs} /$ female). At 22 and $31^{\circ} \mathrm{C}$, females lived longer than the males. The low temperatures affected the reproduction and longevity of $O$. thyestes, suggesting that this species would have better reproductive performance under high temperatures, as it occur in tropical and or subtropical regions.
\end{abstract}

KEYWORDS. Biological control, fecundity, longevity, Orius sp., oviposition.

\begin{abstract}
RESUMO. Influência da temperatura na reprodução e longevidade do predador Orius thyestes Herring (Hemiptera, Anthocoridae). O desenvolvimento e a fecundidade das espécies de Orius são bastante influenciados por uma série de fatores, como as condições ambientais, e em particular, pela temperatura. Esse trabalho teve como objetivo avaliar a reprodução e a longevidade de Orius thyestes Herring 1966 em diferentes temperaturas, tendo como alimento, ovos de Anagasta kuehniella (Zeller, 1879). O experimento foi conduzido em câmaras climatizadas com temperaturas de 16, 19, $22,25,28,31 \pm 1{ }^{\circ} \mathrm{C}$, UR de $70 \pm 10 \%$ e fotofase de 12 horas. Efeito deletério da temperatura em $O$. thyestes foi obtido a $16^{\circ} \mathrm{C}$, na qual apenas $40 \%$ das ninfas atingiram a fase adulta, e destes apenas $19 \%$ não apresentaram deformações morfológicas. O maior período de pré-oviposição foi observado a $19^{\circ} \mathrm{C}(17,8$ dias). Os maiores valores para a fecundidade média total foram registrados a 25 e $28^{\circ} \mathrm{C}$, com 109,2 e 128,2 ovos/fêmea, respectivamente, e o menor a $19^{\circ} \mathrm{C}$, com 22,8 ovos/fêmea. A 22 e $31^{\circ} \mathrm{C}$ as fêmeas viveram mais que os machos, sendo que a $19^{\circ} \mathrm{C}$ a longevidade foi maior, independente do sexo. As baixas temperaturas influenciaram a reprodução e longevidade de $O$. thyestes sugerindo que esta espécie poderá ter melhor performance reprodutiva em temperaturas mais elevadas, como aquelas de regiões tropicais e ou subtropicais.
\end{abstract}

PALAVRAS-CHAVE. Controle biológico, fecundidade, longevidade, Orius sp., oviposição.

A temperatura é um dos principais fatores abióticos que limitam a sobrevivência e reprodução dos insetos (Hallman \& Denlinger, 1998). Com relação aos inimigos naturais, o conhecimento das suas adaptações às condições climáticas tem um papel essencial na obtenção de sucesso em programas de controle biológico. Segundo Bueno (2000) os percevejos predadores do gênero Orius são influenciados pela temperatura, fotoperíodo e alimento, e várias pesquisas evidenciaram que o fotoperíodo e a temperatura exercem efeito direto na atividade reprodutiva desses insetos presentes em regiões temperadas, uma vez que enfrentam o inverno sob formas diapausantes (Kohno, 1998; Nagai \& Yano, 1999). Assim, em condições de baixa temperatura e fotoperíodo curto, as fêmeas paralisam a oviposição, o que provoca uma diminuição nas populações desses predadores comprometendo a sua efetividade como agentes de controle (Alauzet et al., 1994; Kohno, 1998).

O comportamento das espécies originárias da região Paleártica é bem conhecido, entretanto, de acordo com Bueno
(2000) e Lattin (2000) ainda existem poucas informações sobre aquelas presentes em áreas tropicais. A ocorrência de Orius thyestes Herring, 1966, em Lavras, MG e Pindorama, SP, no Brasil, foi registrada pela primeira vez em estudos conduzidos por Silveira et al. (2003) e por se tratar de uma espécie predadora, torna-se importante conhecer as suas várias características biológicas, as quais poderão ser usadas num processo de seleção de agentes de controle biológico. Esse trabalho teve como objetivo avaliar a reprodução e a longevidade de $O$. thyestes em diferentes temperaturas, tendo como alimento ovos de Anagasta kuehniella (Zeller, 1879), visando a sua criação massal e uso em programas de controle biológico.

\section{MATERIAL E MÉTODOS}

O experimento foi conduzido em câmaras climatizadas com temperaturas de $16,19,22,25,28,31 \pm 1^{\circ} \mathrm{C}$, UR de $70 \pm 10 \% \mathrm{e}$ fotofase de 12 horas. Foram utilizados adultos de $O$. thyestes 
recém-emergidos, com até 24 horas, obtidos a partir de ninfas mantidas sob as mesmas condições. A separação dos sexos foi realizada através da análise da genitália, utilizando a metodologia proposta por Tommasini \& Bolkmans (1998).

Os insetos adultos foram individualizados em placas de petri ( $5 \mathrm{~cm}$ de diâmetro) contendo algodão umedecido e ovos de A. kuehniella como alimento. Para o acasalamento, os machos foram mantidos com as fêmeas por 15 minutos/dia no período de pré-oviposição, e por 15 minutos/semana no período de oviposição segundo metodologia proposta por Mendes et al. (2003). Nos recipientes, junto às fêmeas, foi acrescentada uma haste de picão-preto (Bidens pilosa, Linnaeus) (Asteraceae), sem pólen, como substrato de oviposição, envolvida em pedaço de algodão umedecido, trocada diariamente e desinfectada em solução de hipoclorito de sódio $0,5 \%$, por 20 minutos.

O delineamento experimental foi inteiramente casualizado, com seis tratamentos, representados pelas temperaturas e com número diferente de repetições por tratamento. Foram avaliados os períodos de pré-oviposição e oviposição, fecundidade diária e total, longevidade e razão sexual. Também foram determinados o período embrionário e a viabilidade dos ovos nas diferentes temperaturas. Os resultados foram submetidos à análise de variância seguida do teste de agrupamento de médias de Scott \& Knott (1974), a 5\% de probabilidade e complementados com análise de regressão. A temperatura base e constante térmica da fase de ovo foram calculados de acordo com metodologia proposta por Haddad et al. (1999), através do programa MOBAE (Modelos Bioestatísticos Aplicados a Entomologia).

\section{RESULTADOS EDISCUSSÃO}

Foi verificada influência da temperatura nos diferentes parâmetros reprodutivos do predador $O$. thyestes. Na temperatura de $16^{\circ} \mathrm{C}$, apenas $40 \%$ das ninfas atingiram a fase adulta e dessas, somente 19\% apresentaram características morfológicas externas normais. O restante mostrou alterações, como asas deformadas (54\%) e indivíduos que não conseguiram soltar-se totalmente da última exúvia (27\%). Vale ressaltar que a $16^{\circ} \mathrm{C}$ todos os indivíduos, incluindo os adultos normais, permaneceram imóveis nos recipientes, mesmo quando eram colocados para o acasalamento e somente se movimentavam quando eram estimulados mecanicamente com auxílio de um estilete.

Razão sexual. A razão sexual média de $O$. thyestes foi de aproximadamente 0,50 nas temperaturas entre 19 e $28^{\circ} \mathrm{C}$, com tendência para um maior número de fêmeas a $31^{\circ} \mathrm{C}(0,58)$ e maior número de machos a $16^{\circ} \mathrm{C}(0,35)$. Essa baixa produção de fêmeas a $16^{\circ} \mathrm{C}$ pode ser atribuída, provavelmente, às dificuldades encontradas para a visualização dos sexos em $23 \%$ dos indivíduos, devido às alterações morfológicas externas apresentadas pelos indivíduos nessa temperatura. Nas temperaturas de $19,22,25$ e $28^{\circ} \mathrm{C}$ a razão sexual foi de 0,52 ; 0,$53 ; 0,49$ e 0,51 , respectivamente. Ohta (2001) também obteve um maior número de machos de Orius sauteri (Poppius) a 15 ${ }^{\circ} \mathrm{C}$.

Período de pré-oviposição. O período entre a emergência e a primeira oviposição das fêmeas desse predador seguiu um modelo de natureza quadrática (Fig. 1A), reduzindo-se acentuadamente quando a temperatura variou de 19 (17,8 dias) para $25^{\circ} \mathrm{C}$ (5,3 dias) e ocorrendo uma relativa estabilização nesse período quando as fêmeas foram mantidas a 28 e $31^{\circ} \mathrm{C}$, corroborando os resultados de Tommasini (2003) que verificou o menor período de pré-oviposição para Orius laevigatus (Fieber, 1860 ) a $30^{\circ} \mathrm{C}$, comparado com as temperaturas de $14 \mathrm{e}$ $22^{\circ} \mathrm{C}$.

Nas temperaturas mais elevadas, $28 \mathrm{e} 31^{\circ} \mathrm{C}$, o período médio de pré-oviposição de $O$. thyestes foi significativamente menor, comparado com o das demais temperaturas (Tabela I). Alauzet et al. (1994) relataram uma duração de 3 a 4 dias para $O$. laevigatus mantidos à $30^{\circ} \mathrm{C}$, e Nagai \& Yano (1999) também constataram uma menor duração desse período à $30^{\circ} \mathrm{C}$, para $O$. sauteri e $O$. laevigatus.

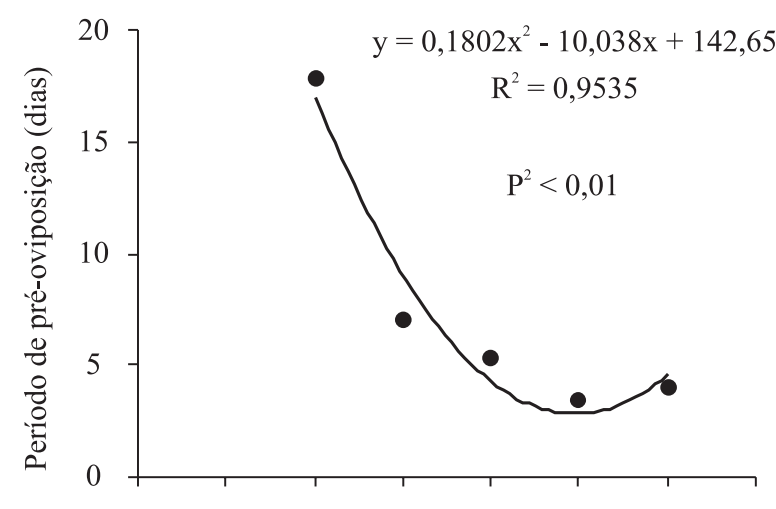

A

Temperatura $\left({ }^{\circ} \mathrm{C}\right)$

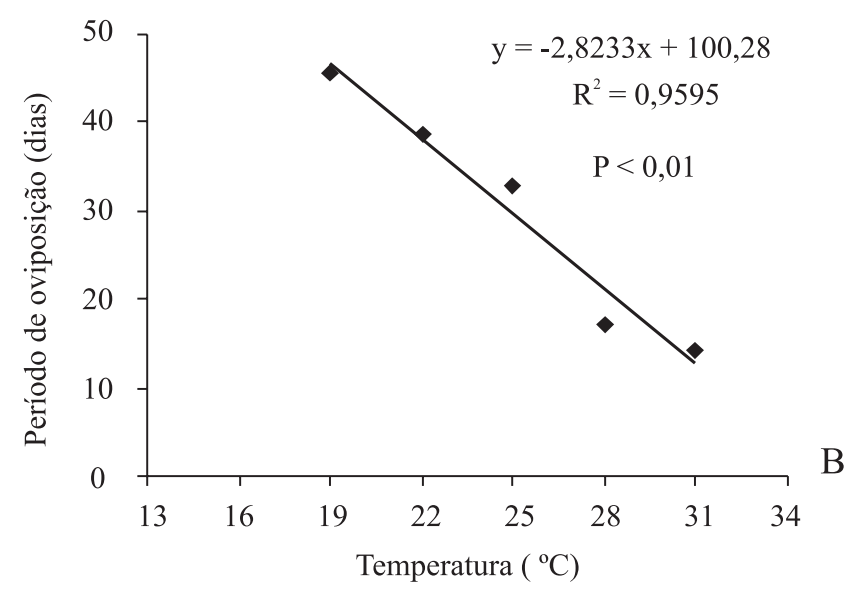

B

Fig. 1. Períodos de pré-oviposição (A) e oviposição (B) de Orius thyestes em diferentes temperaturas, UR de $70 \pm 10 \%$ e fotofase de 12 horas. 
Tabela I. Valores médios ( \pm EP) da duração dos períodos de pré-oviposição e de oviposição e da fecundidade diária e total de Orius thyestes em diferentes temperaturas, UR de $70 \pm 10 \%$ e fotofase de 12 horas.

\begin{tabular}{|c|c|c|c|c|c|c|c|c|}
\hline $\mathrm{T}\left({ }^{\circ} \mathrm{C}\right)$ & $\mathrm{n}$ & $\begin{array}{l}\text { Período de pré- } \\
\text { oviposição }\end{array}$ & $\mathrm{n}$ & $\begin{array}{l}\text { Período de } \\
\text { oviposição }\end{array}$ & $\mathrm{n}$ & $\begin{array}{l}\text { Fecundidade } \\
\text { diária/fềmea }\end{array}$ & $\mathrm{n}$ & $\begin{array}{l}\text { Fecundidade } \\
\text { total/fêmea }\end{array}$ \\
\hline $16^{*}$ & & - & & - & & - & & - \\
\hline 19 & 18 & $17,8 \pm 1,26 \mathrm{a}$ & 18 & $45,8 \pm 3,61 \mathrm{a}$ & 18 & $0,3 \pm 0,07 \mathrm{c}$ & 18 & $22,8 \pm 3,61 \mathrm{c}$ \\
\hline 22 & 25 & $7,0 \pm 0,24 b$ & 25 & $38,7 \pm 1,84 b$ & 25 & $1,4 \pm 0,12 \mathrm{c}$ & 25 & $87,0 \pm 9,21 b$ \\
\hline 25 & 21 & $5,3 \pm 0,29 \mathrm{c}$ & 21 & $32,7 \pm 1,48 \mathrm{c}$ & 21 & $2,9 \pm 0,16 b$ & 21 & $109,2 \pm 11,09 a$ \\
\hline 28 & 30 & $3,5 \pm 0,14 \mathrm{~d}$ & 30 & $17,0 \pm 0,78 \mathrm{~d}$ & 30 & $5,8 \pm 0,30 \mathrm{a}$ & 30 & $128,2 \pm 9,91 \mathrm{a}$ \\
\hline 31 & 23 & $4,1 \pm 0,33 \mathrm{~d}$ & 23 & $14,3 \pm 1,44 \mathrm{~d}$ & 23 & $3,3 \pm 0,38 b$ & 23 & $90,5 \pm 14,71 b$ \\
\hline
\end{tabular}

${ }^{1}$ Médias seguidas de mesma letra na coluna não diferem significativamente entre si, pelo teste de Scott \& Knott (1974), a 5\% de probabilidade.

* Dados insuficientes para análise.

A maior duração do período de pré-oviposição de $O$. thyestes foi observada a $19^{\circ} \mathrm{C}$ com 17,8 dias (Tabela I, Fig. 1A). Nagai \& Yano (1999) relataram que o maior período de préoviposição de $O$. sauteri foi obtido a $15^{\circ} \mathrm{C}$, comparado com as temperaturas de $20,25 \mathrm{e} 30^{\circ} \mathrm{C}$. Segundo alguns autores (Alauzet et al., 1994; Kohno, 1998) ocorre um aumento na duração do período precedente a oviposição nas espécies de Orius em baixas temperaturas.

Os resultados do estudo confirmam que $O$. thyestes a $16^{\circ} \mathrm{C}$ teve dificuldades em manter suas funções vitais sendo desse modo, provavelmente mais adaptado a temperaturas mais elevadas. De acordo com Tommasini (2003), o período de préoviposição é um indicador de como a população de adultos reage a estímulos externos como temperatura e fotoperíodo.

Período de oviposição. O período médio de oviposição de $O$. thyestes foi inversamente proporcional ao aumento da temperatura, ou seja, houve redução do período à medida que a temperatura variou de 19 para $25^{\circ} \mathrm{C}$, com resultados significativamente diferentes entre si e tendência a um modelo linear (Tabela I, Fig. 1B). Entretanto, a duração média da fase reprodutiva tendeu a estabilização nas temperaturas mais elevadas $\left(28\right.$ e $\left.31^{\circ} \mathrm{C}\right), 17,0$ e 14,3 dias, respectivamente. Alauzet et al. (1994), estudando os parâmetros reprodutivos de $O$. laevigatus, relataram uma diminuição no período de oviposição desse predador quando a temperatura variou de 15 para $30^{\circ} \mathrm{C}$.

Fecundidade. A temperatura influenciou diretamente a capacidade reprodutiva de $O$. thyestes. Nas temperaturas de 19 e $22^{\circ} \mathrm{C}$ foi observado que em 18 e $3,8 \%$, das fêmeas, respectivamente, não colocaram ovos. No entanto, o efeito mais evidente da temperatura sobre a fecundidade foi obtido a $16^{\circ} \mathrm{C}$ onde $100 \%$ das fêmeas não ovipositaram. Isso possivelmente é decorrente ao fato de que em temperaturas mais baixas o acasalamento pode ter sido impedido e/ou alterações na fisiologia das fêmeas podem ter ocorrido, uma vez que nas temperaturas mais elevadas $\left(25,28\right.$ e $\left.31^{\circ} \mathrm{C}\right)$ foi verificado que todas ovipositaram. Nagai \& Yano (1999) constataram que a menor porcentagem de oviposição (50\%) das fêmeas de $O$. sauteri ocorreu devido à indução de diapausa reprodutiva ou pela diminuição na atividade de acasalamento provocada pela baixa temperatura $\left(15^{\circ} \mathrm{C}\right)$.
Considerando o padrão de oviposição diário de O. thyestes, foi verificado que o número médio de ovos por fêmea/dia aumentou quando a temperatura variou de 19 para $28^{\circ} \mathrm{C}$. No entanto, na temperatura mais elevada $\left(31^{\circ} \mathrm{C}\right)$ houve uma redução significativa no número médio diário de ovos desse predador (Tabela I, Fig. 2A). Wang et al. (2001) constataram que a oviposição média diária de Orius strigicollis (Poppius) reduziu de 2,0 para 1,6 ovos quando a temperatura variou de 25 para $30^{\circ} \mathrm{C}$. Tommasini (2003) e Nagai \& Yano (1999) relataram que a capacidade de oviposição diária foi proporcional ao aumento da temperatura em O. laevigatus e O. sauteri, respectivamente.

A fecundidade média total de $O$. thyestes também foi influenciada pela temperatura. A 25 e $28^{\circ} \mathrm{C}$ a capacidade de oviposição foi significativamente maior, com uma média total de ovos por fêmea de 109,2 e 128,2, respectivamente (Tabela I, Fig. 2B). Alauzet et al. (1994) constataram que a fecundidade máxima de $O$. laevigatus foi obtida a $25^{\circ} \mathrm{C}$, comparada com as registradas nas temperaturas de 15,20 e $30^{\circ} \mathrm{C}$, respectivamente.

A fecundidade média total de $O$. thyestes a 25 e $28^{\circ} \mathrm{C}$ foi comparável à encontrada para outras espécies de Orius presentes em áreas temperadas, como a de $O$. insidiosus $(130$ ovos) registrada por Malais \& Ravensberg (1992); e as de Orius majusculus (Reuter, 1879), O. laevigatus, Orius niger (Wolff, 1811) e O. insidious, (174,0; 118,6; 54,1 e 144,3 ovos, respectivamente) constatadas por Tommasini (2003).

O menor valor para fecundidade média total por fêmea de O. thyestes ( 22,8 ovos/fêmea) foi observada a $19^{\circ} \mathrm{C}$. Da mesma forma Yano et al. (2002) constataram um menor número de ovos por fêmea de $O$. sauteri $15^{\circ} \mathrm{C}$ e Tommasini (2003) relataram que a capacidade reprodutiva das fêmeas de $O$. laevigatus foi afetada pela baixa temperatura $\left(14^{\circ} \mathrm{C}\right)$, comparado com as temperaturas de 22 e $30^{\circ} \mathrm{C}$.

O número médio de ovos colocados por $O$. thyetes foi, de um modo geral, proporcional ao aumento da temperatura, no intervalo de 19 a $28^{\circ} \mathrm{C}$, e isso, provavelmente, se deve ao aumento da atividade metabólica dos indivíduos causada pelas temperaturas mais elevadas. Entretanto, a $31^{\circ} \mathrm{C}$ apesar da duração média dos períodos de pré-oviposição e oviposição de $O$. thyestes ter sido semelhante àquela de $28^{\circ} \mathrm{C}$, foi observada uma queda em sua fecundidade média diária e total quando as fêmeas foram mantidas a $31^{\circ} \mathrm{C}$ (Tabela I, Fig. 2). Assim, pode- 

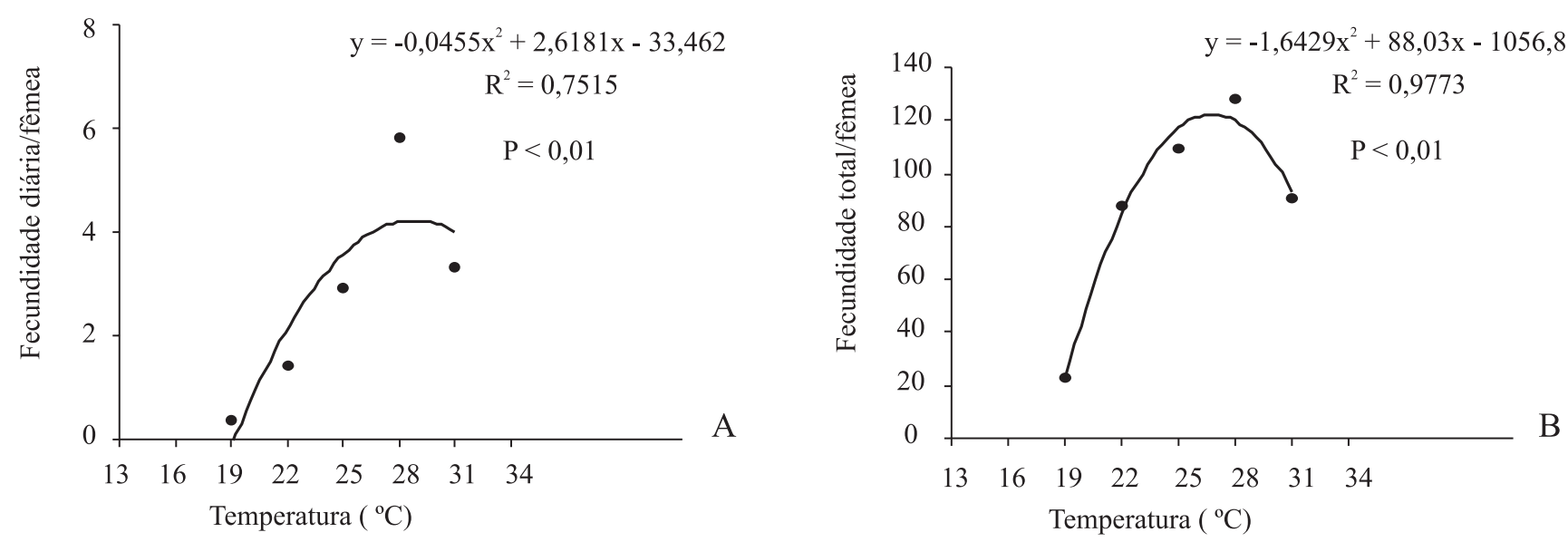

Fig. 2. Fecundidade diária (A) e total (B) de Orius thyestes em diferentes temperaturas, UR de $70 \pm 10 \%$ e fotofase de 12 horas.

se inferir que temperaturas extremas influenciam negativamente os parâmetros reprodutivos desse predador, provavelmente devido ao maior gasto de energia nessas condições. Segundo Ruberson et al. (1998) as temperaturas mais elevadas promovem alta atividade dos predadores, aumentando com isso o gasto da energia armazenada.

Avaliando-se o ritmo de oviposição de $O$. thyestes, foi verificado que aproximadamente $80 \%$ dos ovos produzidos nas temperaturas de $28 \mathrm{e} 31^{\circ} \mathrm{C}$ foram colocados nos primeiros 15 dias após a emergência. Nas temperaturas de 19,22 e $25^{\circ} \mathrm{C}$ a maioria dos ovos produzidos (60, 67 e $74 \%$, respectivamente) foram colocados somente até os 30 dias após a emergência. Esses dados permitem um manejo da temperatura em criação massal para obtenção mais rápida de maior número de indivíduos. Alauzet et al. (1994) observaram que aproximadamente $80 \%$ dos ovos de O. laevigatus foram colocados durante os primeiros 16 dias após o início da oviposição na temperatura de $30^{\circ} \mathrm{C}$, e que $75 \%$ dos ovos foram colocados nos primeiros 29 e 44 dias, respectivamente, a 20 e $25^{\circ} \mathrm{C}$.

Quanto à capacidade reprodutiva de $O$. thyestes, foi observado que o seu melhor desempenho foi nas temperaturas de 25 e $28^{\circ} \mathrm{C}$, mas com fecundidade ligeiramente superior a

Tabela II. Longevidade, em dias $( \pm \mathrm{EP})$, de machos e fêmeas de Orius thyestes em diferentes temperaturas, UR $70 \pm 10 \%$ e fotofase de 12 horas.

\begin{tabular}{ccccc}
\hline $\mathrm{T}\left({ }^{\circ} \mathrm{C}\right)$ & $\mathrm{n}$ & Machos & $\mathrm{n}$ & Fêmeas \\
\hline $16^{*}$ & - & - & - & - \\
19 & 22 & $78,6 \pm 4,40 \mathrm{aA}$ & 18 & $80,5 \pm 5,40 \mathrm{aA}$ \\
22 & 27 & $41,0 \pm 2,50 \mathrm{bB}$ & 25 & $58,7 \pm 5,10 \mathrm{bA}$ \\
25 & 26 & $48,0 \pm 2,80 \mathrm{bA}$ & 21 & $54,0 \pm 4,50 \mathrm{bA}$ \\
28 & 28 & $20,5 \pm 1,13 \mathrm{cA}$ & 30 & $20,9 \pm 1,15 \mathrm{cA}$ \\
31 & 22 & $9,7 \pm 1,40 \mathrm{~dB}$ & 23 & $22,1 \pm 2,65 \mathrm{cA}$ \\
\hline
\end{tabular}

${ }^{1}$ Médias seguidas de mesma letra minúscula na coluna e maiúscula na linha não diferem significativamente entre si, pelo teste de Scott \& Knott (1974), a 5\% de significância. *Dados insuficientes para análise. $28^{\circ} \mathrm{C}$. Além disso, pode-se inferir que essa espécie encontrase adaptada em regiões onde predominam temperaturas mais elevadas, uma vez que as temperaturas mais baixas, no presente estudo não foram favoráveis aos adultos, não ocorrendo oviposição a $16^{\circ} \mathrm{C}$ e esta sendo reduzida a $19^{\circ} \mathrm{C}$.

Longevidade. A longevidade média de $O$. thyestes foi influenciada significativamente pela temperatura, em função dos altos valores de $\mathrm{R}^{2}$ obtidos para ambos os sexos (Fig. 3).

Foi constatado que a $19^{\circ} \mathrm{C}$ a longevidade média foi significativamente maior independente do sexo (Tabela II, Fig. 3 ), confirmando que a longevidade média foi afetada em condições de baixas temperaturas. Já com espécies paleárticas, Nagai \& Yano (1999) verificaram que a maior longevidade de O. sauteri alimentados com Thrips palmi (Karny, 1925) foi obtida a $15^{\circ} \mathrm{C}, 24,9$ e 35,8 dias para machos e fêmeas, respectivamente. Da mesma forma Alauzet et al. (1994) também observaram maior longevidade (70 dias) de O. laevigatus na temperatura de $15^{\circ} \mathrm{C}$.

Nas temperaturas de 22 e $25^{\circ} \mathrm{C}$, não foi verificada diferença significativa na longevidade de machos e fêmeas de $O$. thyestes (Tabela II e Fig. 3). No entanto, a medida em que a temperatura aumentou de 28 para $31^{\circ} \mathrm{C}$, a longevidade dos machos decresceu (Tabela II e Fig. 3A). Nagai \& Yano (1999) também observaram que a longevidade dos machos de $O$. sauteri foi significativamente menor quando mantidos a $30^{\circ} \mathrm{C}(6,6$ dias $)$ do que nas demais temperaturas avaliadas.

Quanto à longevidade das fêmeas de O. thyestes, constatou-se uma tendência à estabilização nas temperaturas de 28 e $31^{\circ} \mathrm{C}$ (Tabela II e Fig. 3B). Esse fato também demonstra uma adaptação às temperaturas mais elevadas, possivelmente devido à ocorrência de um menor gasto de energia na produção de ovos a $31^{\circ} \mathrm{C}$ (Tabela I). Tommasini (2003) mencionou que a longevidade das fêmeas de O. laevigatus reduziu significativamente com o aumento da temperatura. Yano et al. (2002) verificaram que a longevidade das fêmeas de $O$. sauteri foi menor $\left(9,0\right.$ dias) a $30^{\circ} \mathrm{C}$, do que nas temperaturas de 25,20 e $15^{\circ} \mathrm{C}$. 

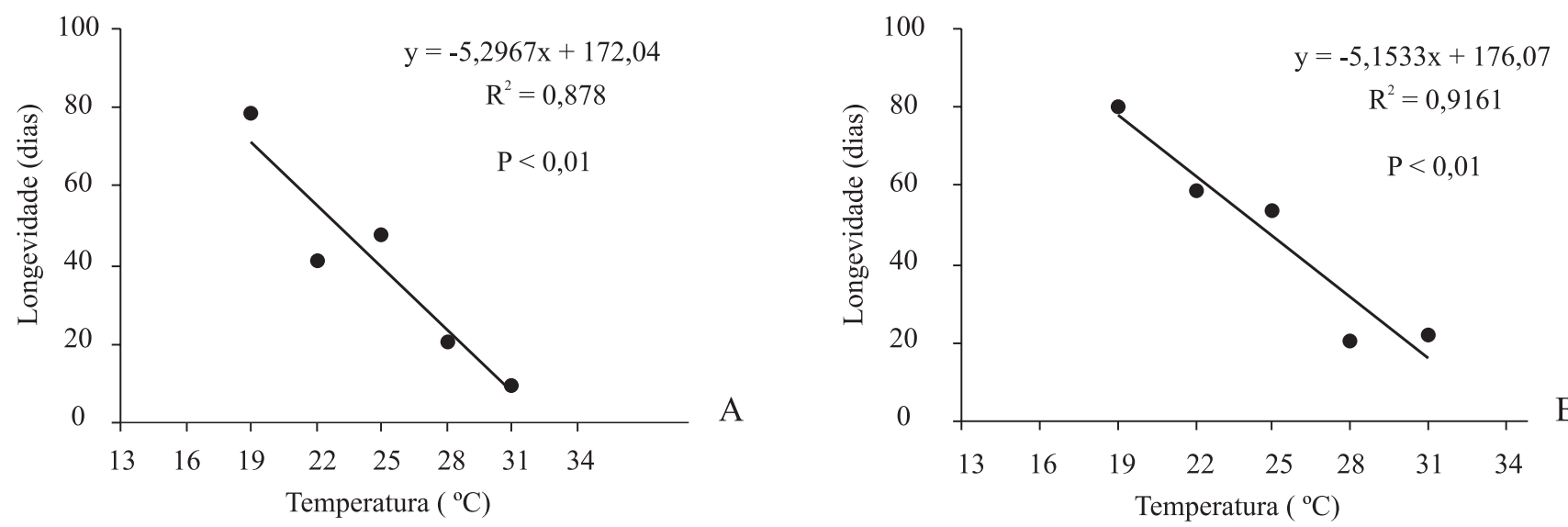

Fig. 3. Longevidade de machos (A) e fêmeas (B) de Orius thyestes em diferentes temperaturas, UR de $70 \pm 10 \%$ de e fotofase de 12 horas.

Houve uma interação significativa da temperatura com a longevidade dos machos e fêmeas de $O$. thyestes quando esses foram mantidos nas temperaturas de 22 e $31^{\circ} \mathrm{C}$ (Tabela II), sendo que as fêmeas viveram significativamente mais tempo do que os machos, em ambas as condições. $\mathrm{O}$ fato das fêmeas terem colocado um menor número de ovos ( 87,0 e 90,5 ovos/fêmea) quando mantidas nas temperaturas de 22 e $31^{\circ} \mathrm{C}$ pode ter contribuído para essa maior longevidade. De acordo com Ruberson et al. (1998), as fêmeas geralmente armazenam maiores teores de lipídios do que os machos, o que pode contribuir para o registro de maiores longevidades entre elas.

Período embrionário. A temperatura teve influência no desenvolvimento dos ovos de $O$. thyestes, o qual seguiu um modelo de natureza quadrática (Fig. 4). Houve uma diminuição significativa na duração média do período embrionário com a elevação da temperatura de 19 a $28^{\circ} \mathrm{C}$ (Tabela III, Fig. 4). Nas temperaturas de 28 e $31^{\circ} \mathrm{C}$ foi observada uma estabilização no desenvolvimento embrionário desse predador, sendo a menor duração registrada a $28^{\circ} \mathrm{C}$, a qual foi significativamente menor que a observada nas demais temperaturas. Resultados semelhantes foram obtidos com outras espécies de Orius

Tabela III. Duração média, em dias $( \pm \mathrm{EP})$, do período embrionário e viabilidade média dos ovos (\%), ( $\pm \mathrm{EP})$ de Orius thyestes em diferentes temperaturas, UR de $70 \pm 10 \%$ e fotofase de 12 horas.

\begin{tabular}{ccccc}
\hline $\mathrm{T}\left({ }^{\circ} \mathrm{C}\right)$ & $\mathrm{n}$ & $\begin{array}{c}\text { Período } \\
\text { embrionário (dias) }\end{array}$ & $\mathrm{n}$ & Viabilidade $(\%)$ \\
\hline $16^{*}$ & - & - & - & - \\
19 & 55 & $11,1 \pm 0,14 \mathrm{a}$ & 55 & $70,0 \pm 8,36 \mathrm{~b}$ \\
22 & 100 & $7,7 \pm 0,16 \mathrm{~b}$ & 100 & $73,3 \pm 8,07 \mathrm{~b}$ \\
25 & 120 & $5,5 \pm 0,14 \mathrm{c}$ & 120 & $86,6 \pm 6,21 \mathrm{a}$ \\
28 & 120 & $3,1 \pm 0,18 \mathrm{~d}$ & 120 & $93,3 \pm 4,55 \mathrm{a}$ \\
31 & 120 & $3,5 \pm 0,14 \mathrm{~d}$ & 120 & $73,3 \pm 8,07 \mathrm{~b}$ \\
\hline
\end{tabular}

${ }^{1}$ Médias seguidas de mesma letra na coluna não diferem significativamente entre si, pelo teste de Scott e Knott (1974), a 5\% de probabilidade. * Dados insuficientes para análise.
(Isenhour \& Yeargan, 1981; Mccaffrey \& Horsburgh, 1986; Malais \& Ravensberg, 1992).

Viabilidade dos ovos. Com o aumento da temperatura de 19 a $28^{\circ} \mathrm{C}$ constatou-se um aumento percentual médio de viabilidade (Tabela III), o qual voltou a cair na temperatura de $31^{\circ} \mathrm{C}$. Os ovos mantidos nas temperaturas mais baixas (19 e $22^{\circ} \mathrm{C}$ ), apresentaram uma viabilidade significativamente semelhante entre si e menor do que aqueles mantidos a $28 \mathrm{e}$ $31^{\circ} \mathrm{C}$ (Tabela III).

Exigências térmicas. A temperatura base $(\mathrm{Tb})$ da fase de ovo de $O$. thyestes foi de $14,62^{\circ} \mathrm{C}$. Tommasini (2003) observou para $O$. laevigatus uma temperatura base de $9,2^{\circ} \mathrm{C}$ e de acordo com Mccaffrey \& Horsburgh (1986) O. insidious apresentou limite térmico inferior de $10,2^{\circ} \mathrm{C}$. Assim, pode-se constatar que $O$. thyestes é mais sensível a temperaturas mais baixas, uma vez que seu limite térmico inferior foi superior aos encontrados para espécies presentes em regiões de clima temperado.

A constante térmica $(\mathrm{K})$ obtida para $O$. thyestes foi de 51,3 graus-dia. Tommasini (2003) constatou uma constante térmica

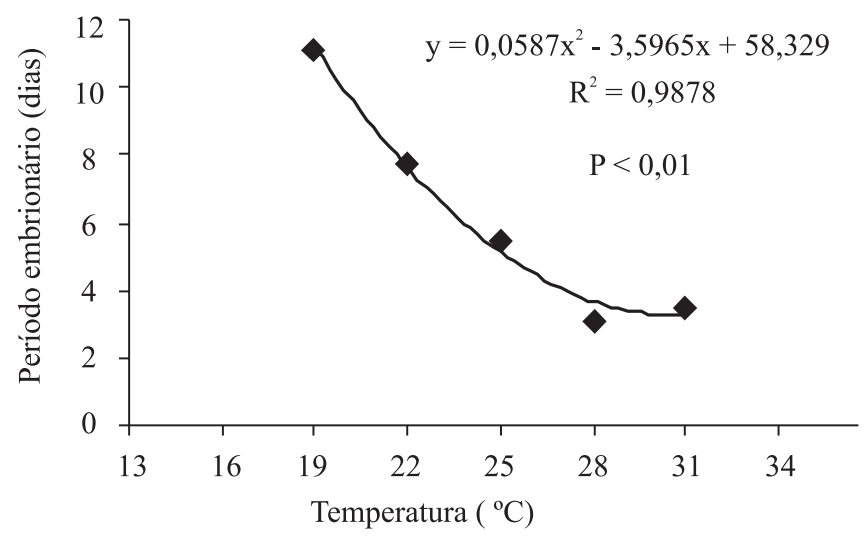

Fig. 4. Período embrionário de Orius thyestes em diferentes temperaturas, UR de $70 \pm 10 \%$ e fotofase de 12 horas. 
de 70,4 graus-dia para O. laevigatus e Mccaffrey \& Horsburgh (1986) relatou 75,8 graus-dia para $O$. insidiosus.

A temperatura foi um fator ecológico muito influente na reprodução e longevidade de $O$. thyestes. Temperaturas mais baixas ocasionaram alterações morfológicas externas, prolongamento do período pré-reprodutivo, reprodutivo e embrionário, além do que algumas fêmeas paralisaram a oviposição e apresentaram menor capacidade reprodutiva. Seu melhor desempenho foi obtido a 25 e $28^{\circ} \mathrm{C}$, podendo ser estas as temperaturas adequadas para a sua criação massal.

Agradecimentos. Ao Conselho Nacional de Desenvolvimento Científico e Tecnológico - CNPq e Coordenação de Aperfeiçoamento de Pessoal de Nível Superior - CAPES pelas bolsas de estudos. A FAPEMIG (Fundação de Amparo a Pesquisa do Estado de Minas Gerais) pelo apoio financeiro. A Dra. Maria Grazia Tommasini (Crop Production Research Centre, Cesena, Italy) pela ajuda quanto à identificação do predador.

\section{REFERÊNCIAS}

Alauzet, C.; D. Dargagnon \& J. C. Malausa. 1994. Bionomics of a polyphagous predator: Orius laevigatus (Heteroptera: Anthocoridae). Entomophaga 39: 3-44.

Bueno, V. H. P. (ed.) 2000. Controle Biológico de pragas: produção massal e controle de qualidade. Lavras, Ed. UFLA, 207p.

Haddad, M. L.; J. R. P. Parra \& R. C. B. Moraes. 1999. Métodos para estimar os limites térmicos inferiores e superiores de desenvolvimento de insetos. Piracicaba: FEALQ, 29p.

Hallman, G. J. \& D. L. Denlinger. 1998. Introduction: Temperature sensitivity and integrated pest management. In: Hallman, G. J. \& D. L. Denlinger. (Ed.). Temperature sensitivity in insects and application in integrated pest management. Boulder: Westview Press. cap. 1. p. 1-5. Disponível em: http:// pestdata.nesc.edu/ipmtext/cap1.pdf> Acesso em 15 ago. 2003.

Isenhour, D. J. \& K. V. Yergan. 1981. Effect of temperature on the development of Orius insidiosus, with note on laboratory rearing. Annals of the Entomological Society of America 74: 114116.

Kohno, K. 1998. Thermal effect on reproductive diapause induction in Orius sauteri (Heteroptera: Anthocoridae). Applied Entomology and Zoology 33: 487-490.
Lattin, J. D. 2000. Economic importance of minute pirate bugs (Anthocoridae). In: Shoefer, C. W. \& A. R. Panizzi. (eds): Heteroptera of economic importance. Florida: CRC Press, 828p.

Maccffrey, J. P. \& L. Horsburgh. 1986. Biology of Orius insidiosus (Heteroptera: Anthocoridae): An predator in Virginia Apple Orchards. Environmental Entomology 15: 984-988.

Malais, M. \& W. J. Ravensberg. 1992. The biology of glasshouse pest and their natural enemies: Knowing and recognizing. Netherlands, Roddernris: Koppert, 109p.

Mendes, S. M.; V. H. P. Bueno \& L. M. Carvalho. 2003. Influence of the presence/ausence of males in the oviposition of Orius insidiosus (Say) (Hemiptera: Anthocoridae). IOBC/WPRS Bulletin 26: $143-146$.

Nagai, D. \& E. Yano. 1999. Effect of temperature on the development and reproduction of Orius sauteri (Poppius) (Heteroptera: Anthocoridae), a predator of Thrips palmi Karny (Thysanoptera: Thripidae). Applied Entomology and Zoology 34: 223-229.

Ohta, I. 2001. Effect of temperature on development of Orius strigicollis (Heteroptera: Anthocoridae) fed on Frankliniella occidentalis (Thysanoptera: Thripidae). Applied Entomology and Zoology 36: 483-488.

Ruberson, J. R.; T. J. Kring \& N. Elkassabany. 1998. Overwintering and the diapause syndrome of predatory Heteroptera. In: Coll, M. \& J. R. Ruberson, Predatory Heteroptera: their ecology and use in Biological control. Entomological Society of America. Lanham, Maryland. p. 49-69.

Scott, A. J.; M. A. Knott. 1974. A cluster analysis method for grouping means in the analysis of variance. Biometrics 30: 507-512.

Silveira, L. C. P.; V. H. P. Bueno \& S. M. Mendes. 2003. Records of two species of Orius Wolff (Hemiptera: Anthocoridae) in Brazil. Revista Brasileira de Entomologia 47: 303-306.

Tommasini, M. G. \& K. Bolckmans. 1998. Orius spp. (Orius laevigatus, Orius insidiosus, Orius majusculus, Orius albidipennis) (Hemiptera: Anthocoridae). Sting, Newsletter on biological Control in Greenhouse. Wageningen, n.18. p.25.

Tommasini, M. G. 2003. Evaluation of Orius species for biological control of Frankliniella occidentalis (Pergande) (Thysanoptera: Thripidae). Wageningen University. (Tesis Doctorate). 215p.

Yano, E.; K. Nagai; K. Watanabe \& K. Yara. 2002. Biological parameters of Orius spp. For control of thrips in Japan. IOBC/ WPRS Bulletin 25: 305-308.

Wang, C. L.; P. C. Lee \& Y. J. Wu. 2001. Field aumentation of Orius strigicollis (Heteroptera: Anthocoridae) for the control of thrips in Taiwan. Food end fertilizer technology center, Taipei, ROC, Exten. Bull. 500, 1-9. Disponível em http//:www.agnet.org/library/ data/eb/eb500/eb500.pdf. (acesso em 25/05/2003).

Recebido em 14.IV.2004; aceito em 11.III.2005 